\title{
Tlx Controls Proliferation and Patterning of Lateral Telencephalic Progenitor Domains
}

\author{
Jan M. Stenman, ${ }^{1,2}$ Bei Wang, ${ }^{1}$ and Kenneth Campbell ${ }^{1}$ \\ ${ }^{1}$ Division of Developmental Biology, Children's Hospital Research Foundation, Cincinnati, Ohio 45229-3039, and 2Wallenberg Neuroscience Center, \\ Division of Neurobiology, Lund University, S-221 84 Lund, Sweden
}

\begin{abstract}
We showed previously that the orphan nuclear receptor $T l x$ is required for the correct establishment of the pallio-subpallial boundary. Loss of Tlx results in a dorsal expansion of ventral markers (e.g., the homeodomain protein GSH2) into the ventralmost pallial region, i.e., the ventral pallium. We also observed a disproportionate reduction in the size of the Tlx mutant lateral ganglionic eminence (LGE) from embryonic day 14.5 onward. Here we show that this reduction is caused, at least in large part, by a proliferation defect. Interestingly, in Tlx mutants, the LGE derivatives are differentially affected. Although the development of the Tlx mutant striatum is compromised, an apparently normal number of olfactory bulb interneurons are observed. Consistent with this observation, we found that $T l x$ is required for the normal establishment of the ventral LGE that gives rise to striatal projection neurons. This domain is reduced by the dorsal and ventral expansion of molecular markers normally confined to progenitor domains flanking the ventral LGE. Finally, we investigated possible genetic interactions between Gsh 2 and $T l x$ in lateral telencephalic development. Our results show that, although Gsh2 and Tlx have additive effects on striatal development, they differentially regulate the establishment of ventral pallial identity.
\end{abstract}

Key words: GSH2; Neurogenin2; NR2E1; striatum; subpallium; tailless; ventral pallium; ventral LGE; dorsal LGE; Dbx1; SFRP2; NKX6.2; Er81; ISL1

\section{Introduction}

The telencephalon is patterned along the dorsoventral and rostrocaudal axes by the action of secreted signaling molecules produced by local signaling centers (Rubenstein et al., 1998; Rallu et al., 2002). This leads to the establishment of discrete progenitor domains marked by the expression of individual, or combinations of, developmental control genes that seem to generate distinct neuronal subtypes (Marin and Rubenstein, 2002; Schuurmans and Guillemot, 2002). For example, the lateral (or intermediate) telencephalon can be subdivided into four progenitor domains: the lateral pallium, ventral pallium, dorsal lateral ganglionic eminence (LGE), and ventral LGE.

The LGE is known from transplantation experiments and genetic studies to generate striatal projection neurons and olfactory bulb interneurons (Deacon et al., 1994; Olsson et al., 1995, 1997, 1998; Wichterle et al., 1999, 2001). Yun et al. (2001) subdivided the LGE ventricular zone (VZ) into separate dorsal and ventral domains on the basis of the expression of the ETS transcription factor Er81 mRNA and high levels of the homeodomaincontaining gene Gsh2 in the dorsal LGE. We then subdivided the LGE subventricular zone (SVZ) on the basis of the expression of Er81 in the dorsal LGE and the LIM homeodomain-containing

Received July 31, 2003; revised Sept. 11, 2003; accepted Sept. 30, 2003.

This work was supported by National Institutes of Health Grant NS044080 and Human Frontiers Science Program Grant RG160-2000B (K.C.). We thank R. T. Yu and R. M. Evans for providing the T/x mice. We gratefully acknowledge the kind gifts of probes and antibodies from D. Anderson, T. Edlund, P. Emson, J. Ericson, M. Götz, T. Jessell, and S. Morton.

Correspondence should be addressed to Kenneth Campbell at the above address. E-mail: kenneth.campbell@chmcc.org.

Copyright $\odot 2003$ Society for Neuroscience $\quad$ 0270-6474/03/2310568-09\$15.00/0 protein Islet1 (ISL1) in the ventral LGE (Stenman et al., 2003a). In addition, we proposed and provided evidence in support of the idea that the ventral LGE generates striatal projection neurons, whereas the dorsal LGE contributes interneurons to the olfactory bulb (Stenman et al., 2003a). The ventral pallium is believed to generate the basolateral nucleus of the amygdala and other structures in the rhinencephalon (Fernandez et al., 1998; Puelles et al., 1999, 2000; Stenman et al., 2003b).

Relatively little is known about the genetic mechanisms that control the establishment and maintenance of these progenitor domains. The establishment of the pallio-subpallial boundary depends on the mutual genetic repression of Gsh 2 and the paired homeodomain-containing gene Pax6 (Corbin et al., 2000; Toresson et al., 2000; Yun et al., 2001). In addition, we showed recently that the orphan nuclear receptor $T l x$ (also known as Tailless, NR2E1), which is broadly expressed in the telencephalon (Monaghan et al., 1995; Stenman et al., 2003b), interacts genetically with Pax6 in the establishment of this boundary (Stenman et al., 2003b). In Tlx mutants, ectopic expression of GSH2 and other ventral genes seem to be restricted to the ventral pallium and could be responsible for the suppression of the ventral pallium markers Dbx1 (Yun et al., 2001) and SFRP2 (secreted frizzledrelated protein 2) (Kim et al., 2001) in this region.

To gain additional insights into the development of lateral telencephalic progenitor domains, we analyzed expression of markers of these domains in Tlx, Gsh2, and Gsh2/Tlx double mutants. We show that $T l x$ and Gsh2, which are known to be involved in striatal development, have additive effects on ventral LGE development. However, although the development of dorsal LGE derivatives appear unaffected in Tlx mutants, high-level 
Gsh2 expression is crucial for the establishment of this domain. In addition, analysis of Gsh2/Tlx double mutant reveals that Gsh2 and $T l x$ differentially regulate ventral pallial development.

\section{Materials and Methods}

Animals and genotyping

Tlx mice and embryos (Yu et al., 2000) were genotyped as described by Stenman et al. (2003b). Gsh2 mice and embryos (Szucsik et al., 1997) were typed by PCR using the following primers: 5Gsh2common, ATGGATGTGTTGGGTTAGACTGGGTTCTGG; 3Gsh2wt, TGCTTCACGCGACGGTTCTGAAACCATATT; and 3Gsh2neo, CAGGACATAGCGTTGGCTACCCGTGATATT. The wild-type allele results in a 317 bp band, whereas the targeted allele gives a 750 bp band. For staging of embryos, the morning of vaginal plug was designated as embryonic day 0.5 (E0.5).

\section{Histological analysis}

Embryos were fixed and sectioned on a cryostat as described previously (Toresson et al., 2000). Immunohistochemistry was performed on slidemounted sections as described by Stenman et al. (2003b). At least three embryos (8-15 sections per embryo) were analyzed with respect to all markers used in this study. Analysis of a total of $>20$ embryos at different embryonic stages showed no significant variation between embryos. Primary antibodies were used at the following concentrations: rabbit antiDARPP-32 (dopamine and cAMP-regulated phosphoprotein) (1:1000; Chemicon, Temecula, CA), rabbit anti-Er81 (1:5000; provided by S. Morton and T. Jessell, Columbia University, New York, NY), rabbit antiGSH2 (1:5000) (Toresson et al., 2000), rabbit anti-ISL1/2 (1:500; provided by T. Edlund, Umeå University, Umeå, Sweden), guinea pig antiNKX6.2 (1:1000; provided by J. Ericson, Karolinska Institute, Stockholm, Sweden) (Vallstedt et al., 2001), rabbit anti-PAX6 (1:200; Covance, Princeton, NJ), and goat anti-PAX6 (1:250; Santa Cruz Biotechnology, Santa Cruz, CA). The secondary antibodies used were biotinylated swine anti-rabbit antibodies (Dako, Carpinteria, CA), biotinylated goat anti-guinea pig antibodies (Vector Laboratories, Burlingame, CA), and biotinylated goat anti-rat antibodies (Vector Laboratories). The $\mathrm{ABC}$ kit (Vector Laboratories) was used to visualize the reaction
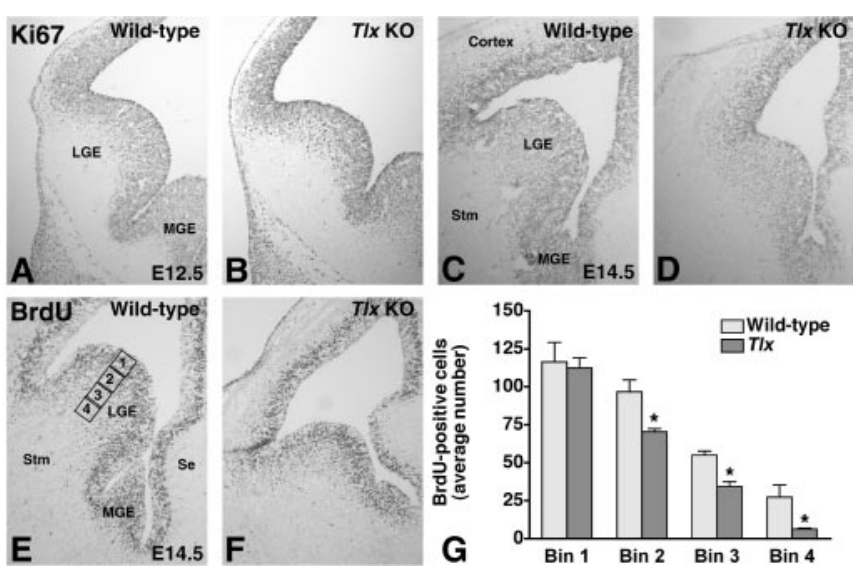

Figure 1. The development of the T/x mutant LGE is compromised. A, C, At E12.5 $(A)$ and E14.5 (C), Ki67, which marks cells in all stages of the cell cycle, is expressed in the LGE VZ and SVZ. $B, A$ A E12.5, there appears to be no significant differences between the $T / x$ mutant and wild-type LGE with respect to size as well as Ki67 expression. D, However, at E14.5, there is both an obvious reduction in size and the number of Ki67-expressing cells in the LGE. E-G, Also, after a $2 \mathrm{hr}$ BrdU pulse, fewer BrdU-positive cells are detected in the E14.5 T/x mutant LGE, particularly in the SVZ. E, We counted BrdU-positive cells in the LGE using four squareshaped bins placed adjacent to each other along the ventriculo-pial axis of the ventral LGE. The first bin extended from the ventricular lumen to the end of the ventricular zone in a wild-type LGE. G, Whereas the number of BrdU-positive cells in the first bin was not significantly different between wild-type and T/x mutant embryos, the number of BrdU-positive cells in the three other bins were. K0, Knock-out; Se, septum; Stm, striatum. ${ }^{*} p \leq 0.01$; Student's unpaired $t$ test between wild-type and $T / x$ mutants.

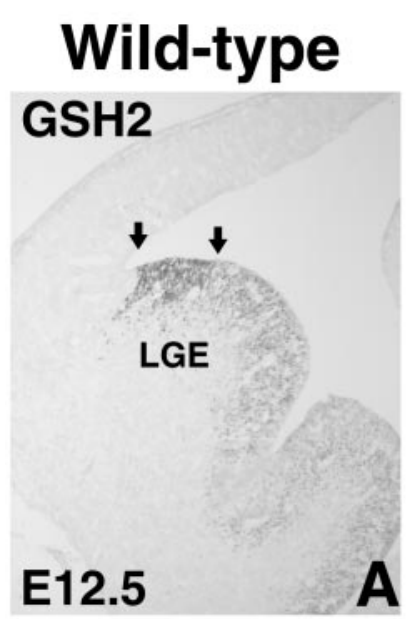

\section{TIx KO}
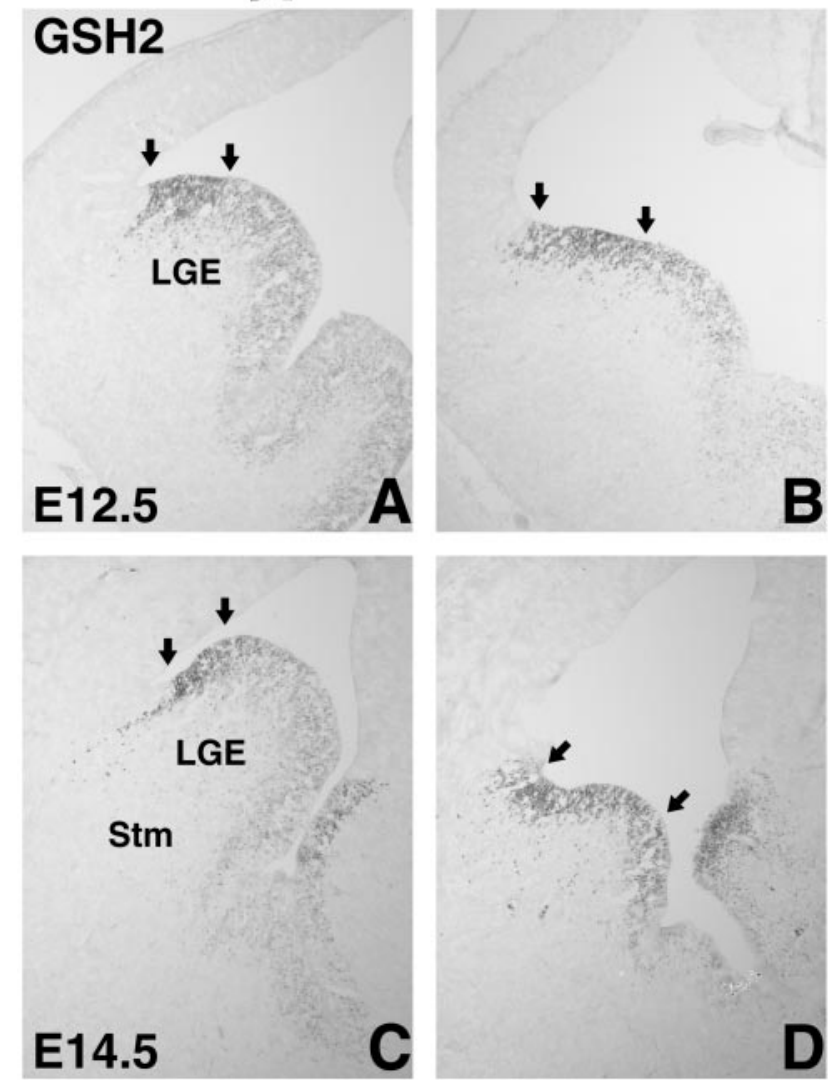

Figure 2. GSH2 expression in the E12.5 and E14.5 T/x mutant LGE. A, C, GSH2 is normally expressed in a gradient throughout the LGE VZ; the highest expression is detected in the most dorsal part of the LGE (indicated by arrows), and the lowest expression is detected in the sulcus between the LGE and MGE. B, D, In T/x mutants, the level of GSH2 expression is more uniform; high levels of GSH2 are detected in a broader region of the LGE VZ (indicated by arrows). In addition, the "thickness" of the expression domain in the VZ is reduced, indicating that the number of precursor cells expressing GSH2 along the ventricular-pial axis is reduced. Stm, Striatum; K0, knock-out.

product for the biotinylated antibodies with diaminobenzidine (Sigma, St. Louis, MO) as the final chromogen.

Nonradioactive in situ hybridization was performed as described by Toresson et al. (1999) using the following probes: $D b x 1$ (I.M.A.G.E. clone 426959), Lhx2 (I.M.A.G.E. clone 2101448), Ngn2 (Sommer et al., 1996), SFRP2 (Kim et al., 2001), and Tbr1 (I.M.A.G.E. clone 303483).

Terminal deoxynucleotidyl transferase-mediated dUTP nick end labeling. Terminal deoxynucleotidyl transferase-mediated dUTP nick end labeling (TUNEL) analysis was performed by first treating the sections with $0.3 \% \mathrm{H}_{2} \mathrm{O}_{2}$ in PBS and subsequently with $0.2 \%$ Triton X-100 for $20 \mathrm{~min}$. The sections were then incubated in the reaction mixture [0.2 $\mathrm{M}$ sodium cacaoylate, $25 \mathrm{~mm}$ Tris-HCl, pH 6.6, $0.25 \mathrm{mg} / \mathrm{ml} \mathrm{BSA}, 1.5 \mathrm{~mm} \mathrm{CoCl}_{2}, 0.4$ $\mu \mathrm{M}$ digoxigenin (DIG)-11-UTP, and $12.5 \mathrm{U}$ of terminal deoxynucleotidyl transferase] for $1 \mathrm{hr}$ at $37^{\circ} \mathrm{C}$ and thereafter incubated with a primary sheep anti-DIG antibody (Roche Diagnostics, Mannheim, Germany) overnight. The secondary antibody used was a biotinylated rabbit antisheep antibody (Vector Laboratories). The ABC kit (Vector Laboratories) was used to visualize the reaction product for the biotinylated antibodies with diaminobenzidine (Sigma) as the final chromogen.

Bromodeoxyuridine labeling. We injected pregnant mice intraperitoneally with bromodeoxyuridine (BrdU) (50-100 mg/kg; Sigma) and subsequently killed the animals $30 \mathrm{~min}$ (E12.5 injection) or $2 \mathrm{hr}$ (E14.5 injection) later. Embryos were processed as described above. Frozen sections were pretreated with $4 \mathrm{~N} \mathrm{HCl}$ for $30 \mathrm{~min}$ at $65^{\circ} \mathrm{C}$ and incubated in normal goat serum for $30 \mathrm{~min}$, after which immunohisto- 
chemistry was performed using a rat antiBrdU antibody (diluted 1:50; Serotec, Oxford, UK).

BrdU-positive cells were counted in four square-shaped bins of equal area that were placed adjacent to each other along the ventriculo-pial axis of the ventral LGE (see Fig. $1 E)$. The first bin stretched from the ventricular lumen to the end of the VZ in a wild-type embryo. We counted a minimum of six sections per embryo from three wild-type and three mutant embryos. We calculated the average number of BrdU-positive cells in each bin for wild-type and Tlx mutant embryos, respectively. We then performed an unpaired $t$ test to determine whether the differences observed were statistically significant ( $p \leq 0.01)$.

\section{Results}

\section{Reduced size of the Tlx mutant LGE}

We noted previously that the E14.5 LGE in Tlx mutants exhibits a disproportionate reduction in size compared with other developing telencephalic structures (Stenman et al., 2003b) (Fig. 1C,D). However, the E12.5 Tlx mutant LGE appears to be relatively normal in size (Fig. $1 A, B$ ). This shows that the dramatic increase in size of the LGE that normally occurs between these stages is compromised. Possible explanations for the observed phenotype are an increase in cell death, a proliferation defect, or a combination of both.

Because no increase in cell death, as detected by TUNEL analysis, is evident at E11.5, E12.5, E13.5, or E14.5 in the $T l x$ mutant telencephalon (data not shown; Roy et al., 2002), we wanted to determine whether a proliferation defect might be responsible for its reduced size. At E12.5, there appears to be no difference in the expression of Ki67 (Fig. 1A,B), which marks cells in all stages of the cell cycle (Schlüter et al., 1993), between wild-type and Tlx mutant LGEs. By E14.5, a reduction in the number of proliferating cells in the Tlx mutant LGE is evident (Fig. 1C,D). Similarly, whereas at E12.5 no obvious difference in the number of BrdU-positive cells was observed after a short pulse (data not shown), at E14.5 the number of cells in S-phase was, as determined by statistical analysis (see Materials and Methods), significantly decreased in the LGE (Fig. 1E, G), particularly in the SVZ (Fig. 1G). Together, these observations indicate that a proliferation defect is primarily responsible for the reduced size of the Tlx mutant LGE. In line with this, expression of GSH2 (Fig. $2 A-D$ ) and MASH1 (mammalian achaete-scute homolog 1) (data not shown) in precursor cells of the Tlx mutant VZ/SVZ shows that the mutant germinal zone appears thinner than in wild types.

The reduction in size of the Tlx mutant LGE at E14.5 suggests that the development of LGE derivatives is compromised in these mutants. A number of studies have shown that the LGE contributes GABAergic interneurons to the olfactory bulb and striatal projection neurons to the developing striatum (Olsson et al.,
TIx KO Gsh2/TIx KO
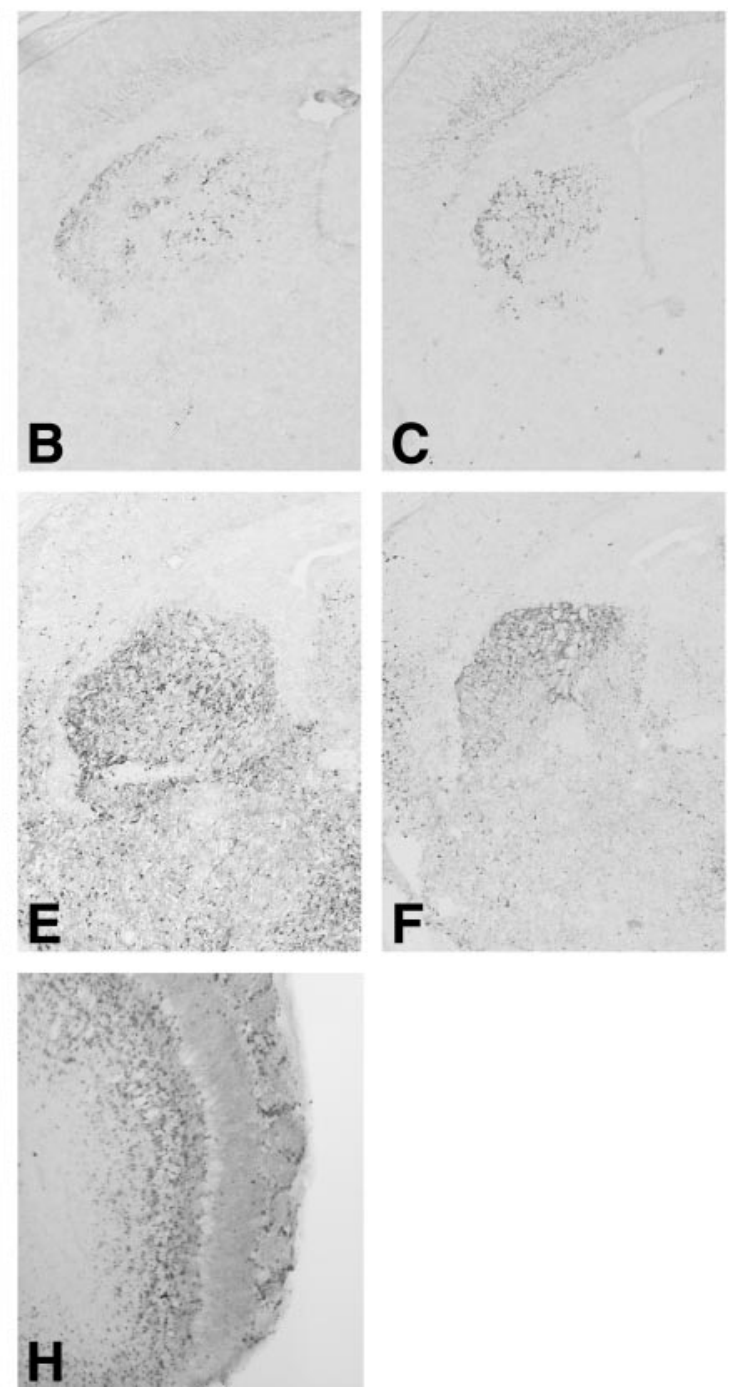

Figure 3. Striatal defects in E18.5 T/x mutant and Gsh2/T/x double-mutant brains. Staining for the striatal markers DARPP-32 $(A-C)$ and calbindin $(D-F)$ shows that the $T / x$ mutant striatum $(S t m ; B, E)$ is smaller than that of a wild-type $(A, D)$, and the Gsh2/TIx double-mutant striatum $(C, F)$ is even smaller. Although the striatum is smaller in each of these mutants, significant lated in the $T / x(E)$ and $G s h 2 / T / x$ mutant striata $(F) . G, H$, There appears to be no significant difference in the number of Er81positive olfactory bulb interneurons in the granule cell $(\mathrm{GCL})$ and mitral and glomerular $(\mathrm{GL})$ layers in the T/x mutant versus wild-type adult brain. ac, Anterior commissure; Iv, lateral ventricle; KO, knock-out.

1997, 1998; Wichterle et al., 2001). We therefore examined the development of these neuronal subtypes in Tlx mutants.

Indeed, at E18.5, the striatum, as marked by DARPP-32 (Fig. $3 A, B)$, which marks the patch compartment at perinatal stages (Foster et al., 1987), and calbindin (Fig. 3C,D), which marks the matrix compartment in the mature striatum (Gerfen et al., 1985), is considerably reduced in size. It should be noted that, at E18.5, Tlx mutant brains are in general smaller than wild-type brains (Monaghan et al., 1997); however, the reduction in size of the Tlx mutant striatum is clearly disproportiate compared with that of other structures, such as the septum and the cerebral cortex (Fig. 3; and data not shown).

In contrast, there appears to be no significant reduction in the number of olfactory bulb interneurons (as marked by Er81) in the Tlx mutants (Fig. 3G,H). Thus, a general reduction in size of the LGE and its progenitor domains does not constitute a satis- 
Wild-type

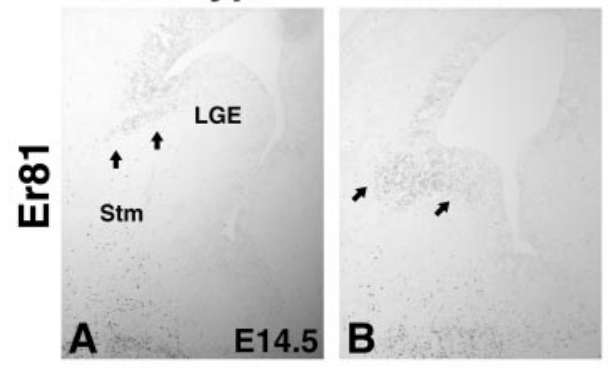

Gsh2 KO Gsh2/TIx KO
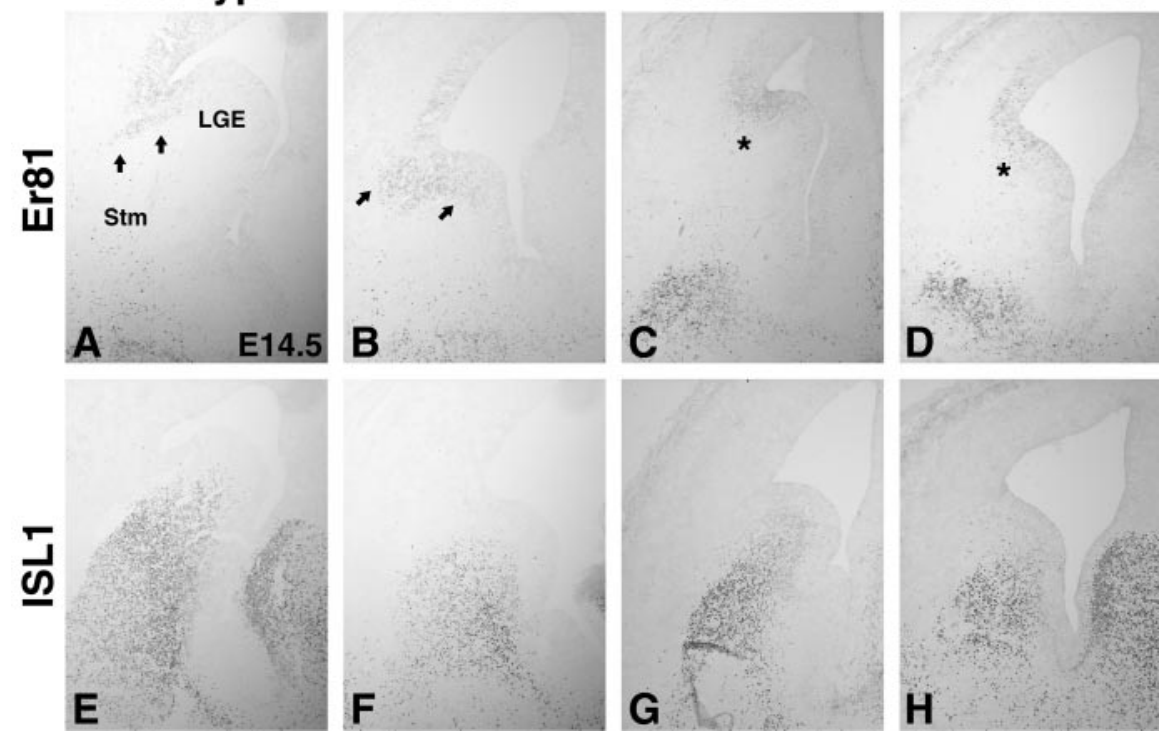

Figure 4. Altered molecular identity in the E14.5 T/x, Gsh2, and Gsh2/T/x mutant LGE. $A$, In the E14.5 wild-type telencephalon, Er81 is expressed in the dorsal LGE SVZ (arrows). In T/x mutants (B), the Er81 expression domain is expanded both dorsally (Stenman et al., 2003b) and ventrally (arrows). Gsh2 mutants ( $C$ and Gsh2/TIx double mutants ( $D$ ) lack SVZ expression of Er81 (C, $D$, asterisks). Note that Er81 expression in the Gsh2 mutants and Gsh2/TIx double mutants is confined to the VZ. E, In the wild type, ISL1 expression is detected in the ventral LGE SVZ and in differentiating cells of the developing striatum (Stm). In T/x mutants $(F)$, the ISL1 expression is weaker and the expression domain is noticably reduced in size at this stage. G, Also, in Gsh2 mutants, fewer ISL1-positive cells are detected and even fewer in the Gsh2/TIX double mutants $(H)$. KO, Knock-out.

factory explanation of the defects observed in Tlx mutant brains. We and others have proposed recently that the LGE is subdivided into a dorsal and a ventral LGE (Yun et al., 2001; Stenman et al., 2003a). We thus wanted to determine whether these progenitor domains are differentially affected in Tlx mutants.

\section{Molecular identity of the dorsal LGE}

Consistent with the preservation of olfactory bulb neurogenesis in Tlx mutants, markers that define the dorsal LGE are still expressed in the LGE (as well as being expanded into the ventral pallium) (Stenman et al., 2003b). Indeed, these even appear to be expanded ventrally. For example, GSH2, which normally is expressed in a gradient (high dorsal, low ventral) within the LGE (Fig. 2A,C), appears to be expressed at high levels throughout much of the Tlx mutant LGE (Fig. $2 B, D$ ). Moreover, Er81 expression in the LGE SVZ is expanded ventrally (Fig. 4B) and appears to overlap with the ISL1 expression domain (Fig. $4 E$ ), unlike the case in wild-types (Fig. $4 A, E$ ). Thus, the expansion of dorsal LGE characteristics in Tlx mutants (Stenman et al., 2003b; data shown here) might be expected to give rise to more olfactory bulb interneurons, which is not the case (Fig. $3 \mathrm{H}$; and data not shown). It should be stressed here, however, that the expansion of dorsal LGE markers does not necessarily imply a respecification of flanking domains (and hence an enlargement of the dorsal LGE domain) but rather most likely represents a misspecification of the progenitor domains. It is also possible that autoregulatory mechanisms exist that control the number of olfactory bulb interneurons that can be generated.

We showed previously that the Er81-positive dorsal LGE is essentially missing in the Gsh2 mutant at E18.5 (Stenman et al., 2003a). This is opposite to that observed in the Tlx mutant (Stenman et al., 2003a) (Fig. 4). Here we show that the dorsal LGE, as marked by Er81 expression in the LGE SVZ, is missing in Gsh2 mutants at E14.5 (Fig. 4C). Together with the expanded domain of high GSH2 expression in Tlx mutants, these data indicate that high levels of GSH2 may be required for the formation of a dorsal LGE. To test the requirement for Gsh2 in dorsal LGE formation, we analyzed Gsh2/Tlx double mutants. Similar to the Gsh2 mutants, these lack Er81 expression in the LGE SVZ (Fig. 4D). Er81 expression in the telencephalon of Gsh2 mutants and Gsh2/Tlx double mutants is confined to the $\mathrm{VZ}$ (Fig. $4 C, D$ ), which is typical of pallial Er81 expression. These findings indicate that Gsh 2 is absolutely required for the correct molecular identity of the dorsal LGE.

\section{Molecular identity of the ventral LGE}

The ventral LGE has been defined by lowlevel expression of GSH2 in the $\mathrm{VZ}$ and by ISL1 expression in the SVZ/mantle zone (MZ) (Yun et al., 2001; Stenman et al., 2003a). In Tlx mutants, the expression domain of the ventral LGE marker ISL1 is drastically reduced in size by E14.5 (Fig. $4 F)$. ISL1 is normally downregulated shortly after birth in the striatal projection neurons but maintains expression in the cholinergic interneurons into adulthood (Wang and Liu, 2001). However, in the Tlx mutants, ISL1 expression is already significantly downregulated at E18.5, unlike that in wild types (data not shown).

The ventral expansion of dorsal LGE characteristics is likely not enough to explain the truncation of the ISL1 expression domain described above. We identified a novel expression domain for the homeobox transcription factor NKX6.2 [also known as GTX (Komuro et al., 1993); antibody described by Vallstedt et al., 2001] in the embryonic telencephalon. NKX6.2 is expressed weakly in the telecephalon as early as E11 (data not shown), but, by E12.5, the expression is strong and confined to the sulcus between the LGE and medial ganglionic eminence (MGE) (Fig. $5 A$ ). It remains expressed in this region at all prenatal stages (Fig. $5 C$, shown at E14.5; and data not shown). This telencephalic expression pattern has been confirmed by analysis of heterozygote embryos in which the $N k x 6.2$ coding region has been replaced with the tau-lacZ gene [J. Ericson (Karolinska Institute, Stockholm, Sweden), personal communication; mice described by Vallstedt et al., 2001]. In E12.5 Tlx mutants, ectopic NKX6.2 expression is observed within the VZ of the ventral LGE (Fig. 5B). At E14.5, strong NKX6.2 expression marks only a small domain (Fig. 5D); however, as mentioned above, the size of the entire mutant LGE is considerably smaller at this stage than in wild-type embryos (Fig. 5C). In addition, scattered NKX6.2-expressing cells can be detected throughout most of the mutant LGE VZ (Fig. 5D, inset). No obvious change in the expression of the MGE markers NKX2.1, Lhx6, Lhx6.1, or $L h x 7$ is observed in the Tlx mutant telencephalon (data not shown). However, the expression of NKX6.2 is expanded into the MGE of the Tlx mutants (Fig. 5B). This shows that the NKX6.2-positive/NKX2.1-negative domain is distinct from the NKX2.1-expressing MGE in its regulation by $T l x$ and that MGE development is relatively unperturbed in Tlx mutants. Together, the dorsal expansion of the NKX6.2 expression domain and the ventral expansion of the Er81 expression domain show that the ventral LGE of Tlx mutants is 
misspecified by the ectopic expression of genes normally flanking its borders.

In addition to having a partially mispatterned LGE VZ, E12.5 Gsh2 mutants lack, or at least have a severely compromised, LGE SVZ, and, hence, generation of early-born striatal projection neurons is compromised (Corbin et al., 2000; Toresson et al., 2000; Toresson and Campbell, 2001; Yun et al., 2001). Later on, a recovery, mediated by the expansion of Gsh1, occurs and later aspects of striatal neurogenesis appear to be carried out (Corbin et al., 2000; Toresson et al., 2000; Toresson and Campbell, 2001; Yun et al., 2001). Tlx mutants, on the other hand, do not exhibit such a recovery, nor do they show ectopic Gsh 1 expression in the LGE at E14.5 (data not shown). Hence, a reasonable assumption is that Gsh2/Tlx double mutants would exhibit additive defects on striatal development. Consistent with this prediction, the E18.5 Gsh2/Tlx double-mutant striatum is greatly reduced in size (Fig. $3 C, F$ ) compared with that of a wild type (Fig. $3 A, D$ ); in addition, it is also smaller than that of a Tlx mutant and Gsh 2 mutant (Fig. $3 B, E$; and data not shown). Like in the Gsh2 (Toresson and Campbell, 2001) and Tlx mutants (Fig. $3 E$ ), calbindin appears to be expressed at higher levels in the double mutants (Fig. $3 F$ ). Although the double-mutant striatum is smaller, significant DARPP-32 expression is observed in the developing striatum (Fig. 3C). This result is opposite to that observed for DARPP-32 expression in Gsh 2 mutants. Interestingly, as in Tlx mutants, the level of ISL1 expression in Gsh2/Tlx double mutants is lower (data not shown).

The worsening of the striatal phenotype in the Gsh2/Tlx double mutants suggests that the development of the ventral LGE would be even more severely affected than in either single mutant. Previous work has shown that the ventral LGE, as marked by ISL1 expression, is truncated in the Gsh 2 mutant (Fig. 4G) (Toresson et al., 2000; Toresson and Campbell, 2001). This truncation has been seen primarily as a result of ectopic PAX6 expression in the dorsalmost LGE. Surprisingly however, the Gsh2 mutant LGE also exhibits ectopic expression of NKX6.2 in scattered cells located throughout most of the LGE VZ (Fig. $6 \mathrm{H}$, inset). As demonstrated above, the ventral LGE is also truncated in Tlx mutants. In fact, at E14.5, the ISL1 expression domain in both Tlx (Fig. 4F) and Gsh2/Tlx mutants (Fig. $4 H$ ) is even smaller than that in Gsh2 mutants (Fig. $4 G$ ). Interestingly, the patterning defects observed in the E12.5 Gsh2/Tlx double-mutant LGE appears to reflect both the Gsh 2 and Tlx phenotypes. NKX6.2 expression is ectopic in the ventral part of the LGE (Fig. $6 C$ ), as is the case in the Tlx mutants (Fig. $5 B$ ), and PAX6 is expanded into the dorsal half of the LGE (Fig. $6 F$ ), similar to that in Gsh 2 mutants (Fig. $6 E$ ). Interestingly, the PAX6-positive cells that emanate from the pallio-subpallial boundary appear to be similar in number between both Gsh2/Tlx double mutants (Fig. 6F) and wild types (Fig. 6D). This is despite the fact that these cells are severely depleted in Gsh2 mutants (Fig. 6E) (Toresson et al., 2000).

At E14.5, the Gsh1-dependent recovery has begun in Gsh2 mutants (Corbin et al., 2000; Toresson et al., 2000; Toresson and Campbell, 2001; Yun et al., 2001) (Fig. 6, compare J, K). As in Gsh2 mutants (Fig. 6K), PAX6 expression in Gsh2/Tlx double mutants has retracted to the LGE-pallium angle (Fig. $6 \mathrm{~L}$ ), similar to that in wild types (Fig. $6 \mathrm{~J}$ ). In addition, Gsh1 is, as in $G s h 2$ mutants, ectopically expressed throughout the LGE (data not shown). In contrast to single Tlx or Gsh2 mutants, NKX6.2 expression is significantly upregulated throughout the E14.5 double-mutant LGE (Fig. 6I). The expression domain of NKX6.2 now nearly abuts that of PAX6, suggesting that most of the LGE $\mathrm{VZ}$ is misspecified in the double mutants. This expansion of the

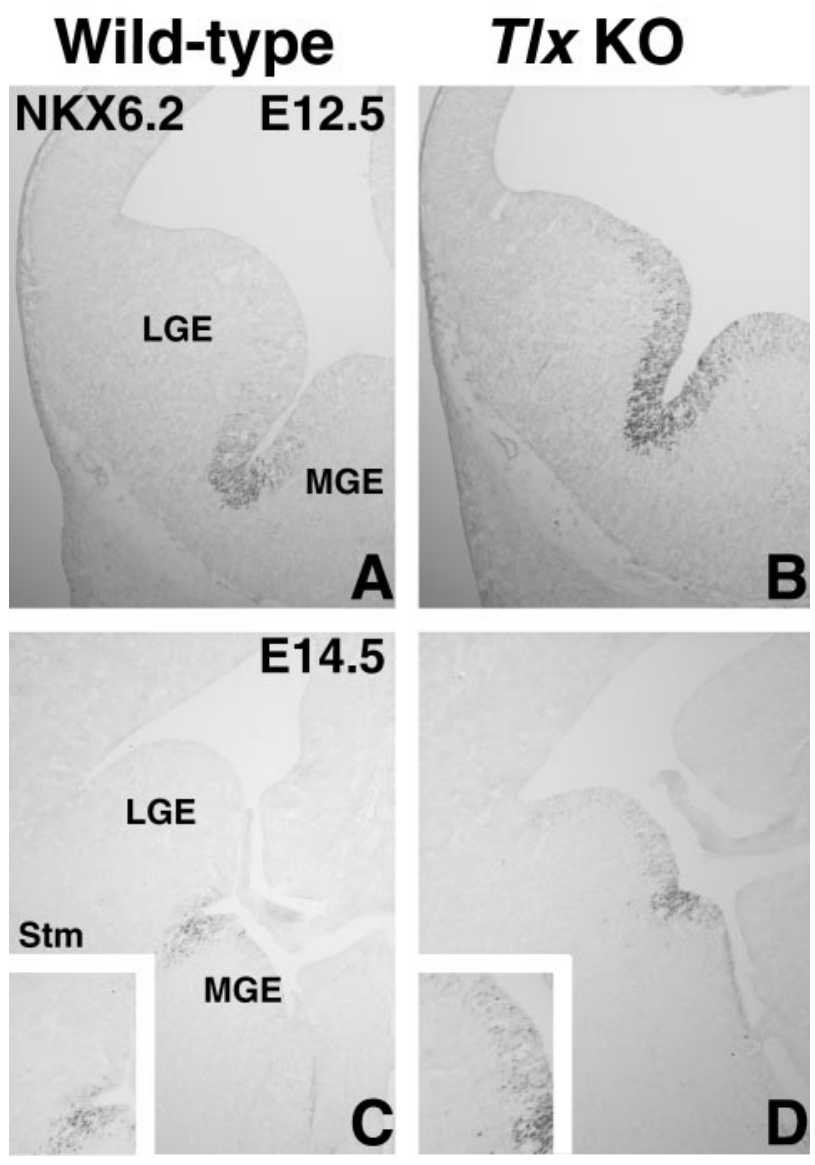

Figure 5. NKX6.2 expression in wild-type and T/x mutant embryos. A, At E12.5, NKX6.2 is expressed in the sulcus between the LGE and MGE. A few scattered cells can also be detected in the LGE and MGE VZ right next to the domain of high NKX6.2 expression. B, In the T/x mutant, NKX6.2 is ectopically expressed in the ventral half of the LGE. C, Also at E14.5, NKX6.2 expression is detected in the VZ between the LGE and MGE. D, In E14.5 T/x mutants, the NKX6.2 expression domain in the mutants appears rather small; however, considering that the LGE is smaller in the mutant at this stage, the domain is relatively larger. In addition, NKX6.2 is ectopically expressed in scattered cells throughout much of the LGE VZ (compare insets in C and D). KO, Knock-out; Stm, striatum.

NKX6.2 expression domains occurs, as in Tlx mutants, without an enlargement of the NKX2.1 expression domain (data not shown).

\section{Molecular identity of the ventral pallium}

We showed previously that GSH2 is ectopically expressed in the most ventral part of the $T l x$ mutant pallium and that this region lacks expression of the ventral pallium markers Dbx1 and SFRP2 (Stenman et al., 2003b). Because Gsh2 is required to repress $D b x 1$ expression in the subpallium (Yun et al., 2001), it may be that its dorsal expansion in the Tlx mutant telencephalon is responsible for the loss of ventral pallial identity (Stenman et al., 2003b). In E12.5 Gsh2 mutants, $D b x 1$ is ectopically expressed throughout the LGE (Fig. 7B) (Yun et al., 2001). Dbx1 is also expressed throughout the LGE in E12.5 Gsh2/Tlx double mutants (Fig. 7C); however, fewer expressing cells are observed than in Gsh $2 \mathrm{mu}-$ tants. Thus, $T l x$ seems to be required for the robust expression of $D b x 1$ in the lateral telencephalon, whereas Gsh2 functions to restrict its expression to the ventral pallium. The expression domain of SFRP2, on the other hand, appears to require Tlx for its expression in the lateral telencephalon (Fig. $7 F$ ). The expression of this gene is not dependent on Gsh2; however, its expression 
shifts ventrally into the LGE (Fig. 7E), corresponding to the ventral limit of PAX6 expression (Fig. 6E). By E14.5, when the recovery has occurred in the Gsh2 mutant LGE, SFRP2 expression is found at the LGE-pallium angle, similar to that in wild types (data not shown). In addition, Ngn2, which is missing from the ventralmost part of the pallium in Tlx mutants (Stenman et al., 2003 b), is expanded ventrally into the dorsal half of the LGE in the Gsh2/Tlx double mutants, similar to that in Gsh2 mutants (data not shown; Corbin et al., 2000; Toresson et al., 2000; Yun et al., 2001), suggesting that Gsh2 negatively regulates Ngn2 expression. Despite these molecular alterations, the expression of Tbr1, a pallial marker, and $L h x 2$, a marker of the ventral pallial MZ, can still be detected in the lateral telencephalon (Fig. $8 A-D$ ). Therefore, although our data show that $T l x$ and Gsh2 differentially regulate the molecular identity of the ventral pallium, aspects of ventral pallial identity can develop in the absence of these gene products.

\section{Discussion}

\section{Telencephalic progenitor domains}

In the ventral spinal cord, progenitor domains for distinct neuronal subtypes have been well characterized on the basis of restricted expression of developmental control genes (for review, see Jessell, 2000; Briscoe and Ericson, 2001). Cross-repressive actions between these developmental control genes have been shown to be required for the refinement of progenitor domains along the dorsoventral axis (Muhr et al., 2001; Novitch et al., 2001; Vallstedt et al., 2001). It is now clear that the telencephalic neuroepithelium also can be divided into a number of molecularly distinct progenitor domains, including those studied here: the ventral pallium, dorsal LGE, and ventral LGE (for review, see Marin and Rubenstein, 2002).

In this study, we reported a novel expression domain for the homeobox transcription factor NKX6.2. Cells expressing this protein are found in the sulcus between the LGE and MGE. This interganglionic expression of NKX6.2 may mark a new progenitor domain in the telencephalon. We currently do not know what cell types derive from this domain. In the spinal cord, NKX6.2 plays an important role in establishing the boundary between the most dorsal progenitor domains in the ventral spinal cord, $\mathrm{p} 0$ and p1; the establishment of these progenitor domains depends crucially on cross-repression with Dbx1 (Vallstedt et al., 2001). It is interesting to note that the telencephalic expression of NKX6.2 and GSH2 appears to be complementary (data not shown). It is therefore possible that NKX6.2 acts as a repressor also in the telencephalon and regulates the establishment of the ventral expression border of the ventral LGE progenitor domain.

Information remains scarce as to how the gene expression boundaries between the defined progenitor domains are established in the developing telencephalon. Moreover, it is quite likely that a number of domains have yet to be identified. In addition, a detailed analysis of what neuronal subtypes are generated by each progenitor domain is still lacking. In this study, we contributed to the understanding of how the gene expression boundaries marking distinct progenitor domains both within and flanking the LGE are established.

\section{Control of ventral LGE development}

In a recent study examining the $T l x$ mutant cortex, Roy et al. (2002) provided evidence suggesting that mutant cortical cells undergo precocious differentiation and, as a result, deplete the progenitor pool midway through cortical neurogenesis. Moreover, their results from BrdU-incorporation studies indicate that, between E9 and E14, the cell cycle of cortical progenitors is shortened in Tlx mutants compared with wild types. Together, these defects were proposed to account for the observed thinning of the Tlx mutant cortex. Although the entire telencephalon of Tlx mutants is reduced compared with wild types (Monaghan et al., 1997), the LGE and, importantly, only one of its derivatives, the striatum, are proportionately more affected than other telencephalic regions. Therefore, the proliferation defect we reported here, and which was described previously for the cortex (Roy et al., 2002), is unlikely to account for all of the defects observed in the ventral telencephalon but is probably only an element of a more complex phenotype.

Previous studies have shown that ISL1 marks differentiating striatal projection neurons but is rapidly downregulated after birth (Toresson et al., 2000; Toresson and Campbell, 2001; Wang and Liu, 2001). Our present findings demonstrate that ISL1 is already downregulated by E18.5 in Tlx mutants. This finding would support the precocious differentiation hypothesis of Roy et al. (2002); however, the ISL1-expressing ventral LGE is already reduced in size by E14.5, indicating that even early striatal projection neuron generation is compromised. This reduction in size of the ventral LGE is observed simultaneously with that of a ventral expansion of dorsal LGE markers and a dorsal expansion of the interganglionic marker NKX6.2. This indicates that both the proliferation and the patterning defects are early events that occur at the earliest steps of LGE development. It is possible that either the proliferation defect causes the patterning defect or the other way around. In any case, the disproportionate reduction in the ventral LGE and subsequently the striatum is likely to result from the additive effects of both the proliferation and patterning defects.

In Gsh2 mutants, the dorsal LGE is most severely reduced; however, a significant truncation of the ventral LGE also occurs (Yun et al., 2001; Stenman et al., 2003a). The Tlx phenotype, on the other hand, is most severe in the ventral LGE. Thus, when removing both $T l x$ and Gsh2, the effect on ventral LGE, and therefore on the striatum, is even more severe than in either of the single mutants. Whereas, at early stages (i.e., E12.5) in Gsh2/Tlx double mutants, PAX6 is ectopically expressed in the dorsal half of the LGE, NKX6.2 is ectopically expressed in the ventral half; this leaves only a small domain, which is, at least apparently, correctly patterned. However, by E14.5, the expression domain of NKX6.2 and PAX6 nearly abut in the LGE-cortex angle of the double mutants. This suggests that very few striatal projection neurons would be generated during late stages of striatal development. Moreover, because Gsh 2 mutants lack the early formation of the LGE SVZ, it is likely that both early and late striatal neurogenesis is affected in the Gsh $2 /$ Tlx mutants (Toresson et al., 2000; Yun et al., 2001).

\section{Control of dorsal LGE identity}

As mentioned above, Gsh2 gene function is crucial for the expression of dorsal LGE characteristics. It is interesting to note that high-level GSH2 expression is observed in more ventral regions of the Tlx mutant LGE, suggesting that $T l x$ is required to confine high-level GSH2 expression to the dorsal LGE. This concept is hard to reconcile with the observation that GSH2 and Tlx are both expressed at high levels in the dorsal LGE. However, it is clear that, together with $P a x 6, T l x$ regulates a genetic program that represses Gsh2 from the ventral pallium (Stenman et al., 2003b). Thus, there might be a specific genetic partner for Tlx in the ventral LGE. It may also be that $T l x$ represents a weak repressor of Gsh 2 and that there is a patterning center in the lateral 
telencephalon that induces high levels of Gsh2 by virtue of it close proximity.

Er81 gene expression is found in both the VZ and SVZ of the dorsal LGE (Yun et al., 2001); however, expression of the protein is confined of the SVZ of the dorsal LGE (Stenman et al., 2003a). It is interesting to note that the expression of Er81 in the SVZ correlates well with high levels of $\mathrm{GSH} 2$ in the overlying VZ. Indeed, the restriction of Er81 to the SVZ of the dorsal LGE is dependent on Gsh2 gene function. In both Gsh 2 mutants and Gsh2/Tlx double mutants, Er81 is expressed in the LGE $\mathrm{VZ}$ and not in the SVZ.

\section{Control of ventral pallial identity}

We suggested previously that Tlx might be required for driving expression of $\mathrm{Ngn} 2$ in the ventral pallium (Stenman et al., 2003b). However, at E12.5, in the Gsh2/Tlx double mutants, both PAX6 and Ngn2 are, as in Gsh2 mutants, ectopically expressed in the dorsal half of the LGE, and, at E14.5, after the Gsh1-mediated recovery (Toresson and Campbell, 2001), PAX6 and Ngn2 are expressed in their normal expression domain. This indicates that the ectopic expression of GSH2 in the ventral pallium of Tlx mutants is responsible for the repression of Ngn2. This does not preclude the possibility that Tlx may also promote Ngn2 expression in the ventral pallium; however, this effect would clearly be less significant than the repressive effect of Gsh2. Dbx1 regulation in the lateral telencephalon is more complicated. Analysis of Gsh2 mutants has shown that Gsh2 genetically represses $D b x 1$ expression (Yun et al., 2001; this study). However, in Gsh2/ Tlx double mutants, $D b \times 1$ is expressed at lower levels than in Gsh2 mutants, showing that $T l x$ expression is required for high levels of $D b \times 1$ expression in the telencephalon. SFRP2 appears to be regulated in a more simple manner. No evidence exists in support of Gsh2 being a repressor of SFRP2. In fact, SFRP2 is expressed at both E12.5 and E14.5 in Gsh2 mutants; the expression domain is shifted at E12.5 to the most ventral part of the PAX6 expression domain, whereas at E14.5 SFRP2 expression is indistinguishable from that in wild types. SFRP2, however, is missing in Sey mutants (Kim et al., 2001) as well as in Tlx mutants (Stenman et al., 2003b) and Gsh2/ Tlx mutants. Thus, Pax6 and Tlx could be direct regulators of SFRP2. At least one allele of both Pax6 and Tlx is absolutely required for the lateral telencephalic expression of SFRP2 (Stenman et al., 2003b). In summary, whereas Gsh2 appears to be a negative regulator of ventral pallial iden-

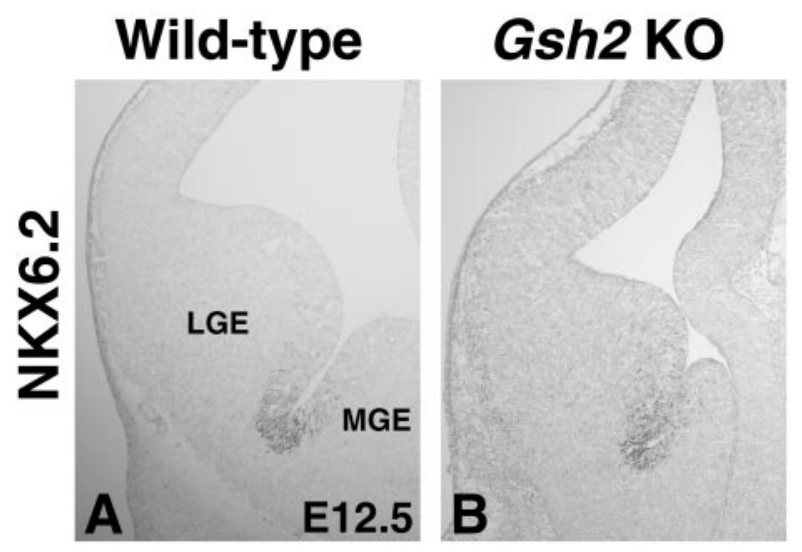

Gsh2/TIx KO
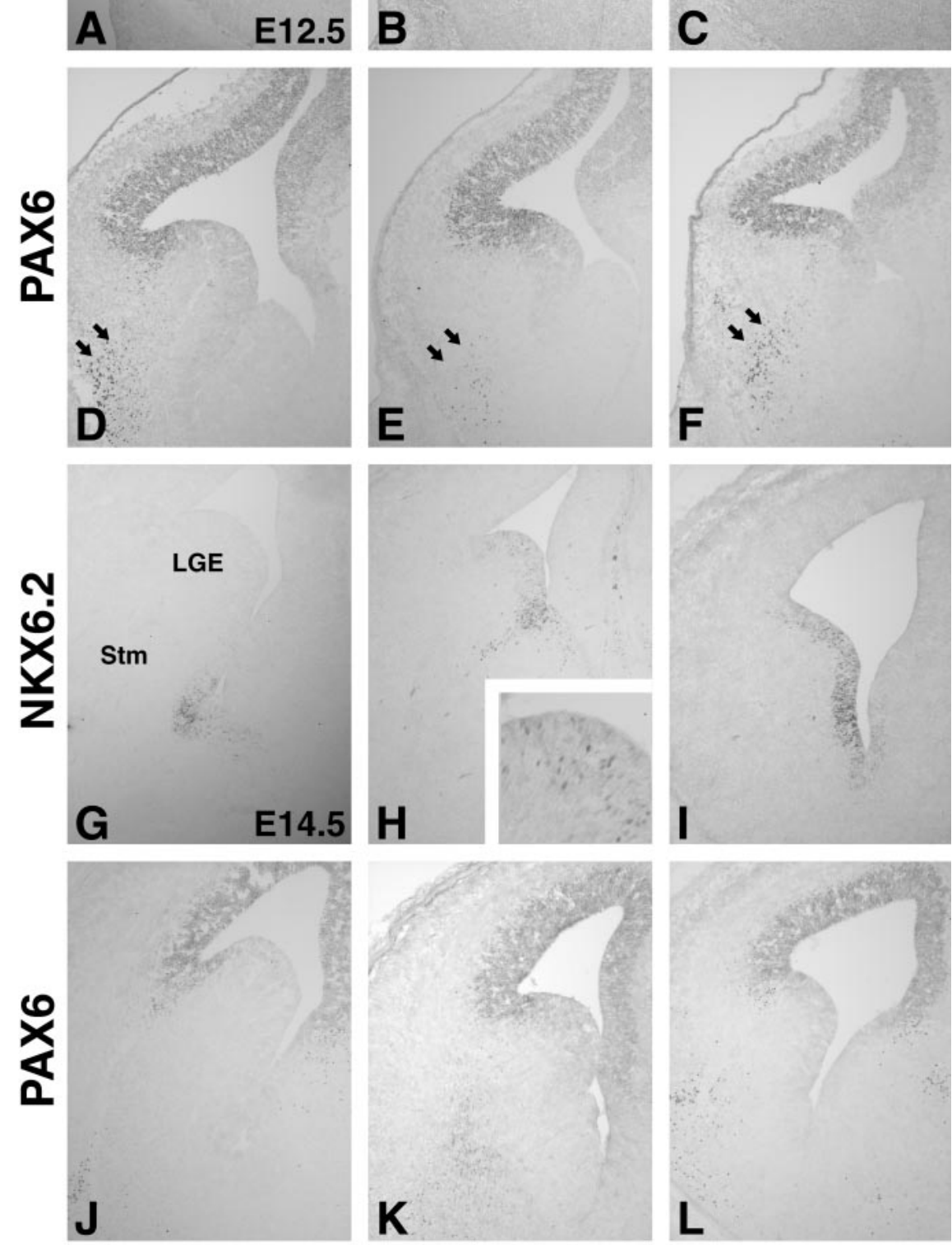

Figure 6. Patterning defects in Gsh2 mutants and Gsh2/Tlx double mutants. A, At E12.5, NKX6.2 is expressed in the sulcus between the LGE and MGE. B, In Gsh2 mutants, a number of scattered cells expressing NKX6.2 ectopically are detected in the ventral most part of the LGE. Note also that the size of the mutant LGE is smaller than the wild-type LGE. C, In the Gsh2/T/x double mutants, as in the T/x mutants (Fig. 2B), NKX6.2 is ectopically expressed in the ventral half of the LGE. D, High PAX6 expression normally wraps around the pallio-LGE angle and stops in the dorsalmost portion of the LGE. In addition, a stream of PAX6-positive cells emanates from the ventralmost region of the PAX6 VZ expression domain down to the mantle zone (arrows). E, In Gsh2 mutants, PAX6 is ectopically expressed in the dorsal half of the LGE. Interestingly, the number of PAX6-positive cells in the mantle zone (arrows) is significantly reduced in these mutants. F, PAX6 is ectopically expressed also in the dorsal half of the Gsh2/TIx double-mutant LGE. Surprisingly, in the Gsh2/T/x double mutants, there appears to be a considerable number of PAX6-positive cells in the mantle zone (arrows). Note also that the ectopic expression of PAX6 and NKX6.2 in the double-mutant LGE leaves only a small domain of the ventral LGE correctly patterned $(C, F) . G$, NKX6.2 expression at E14.5 in the wild-type telencephalon. $H$, 


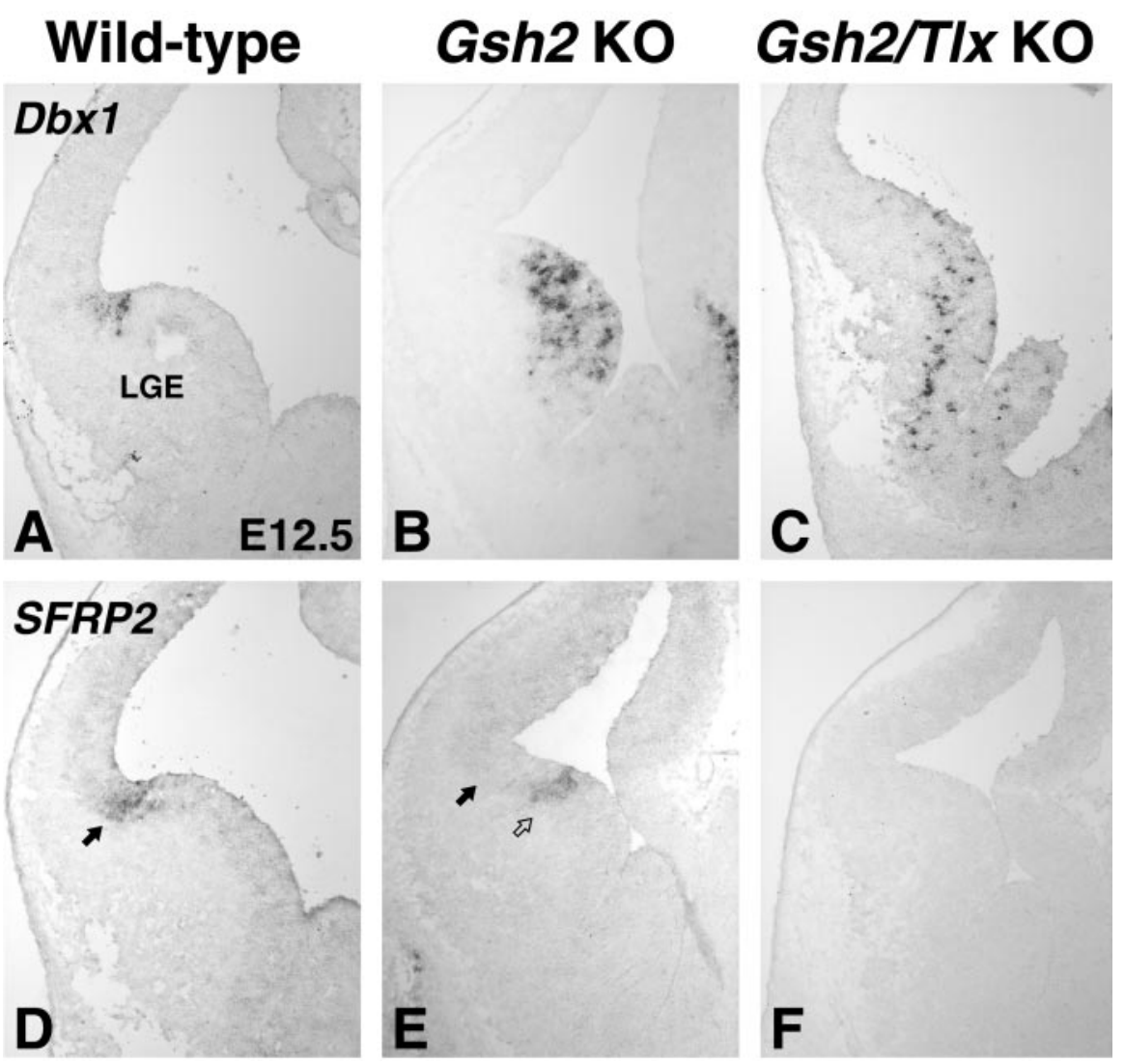

Figure 7. Genetic regulation of ventral pallial identity. $A, D b x 1$ is a marker of the ventral pallium. $B$, In the Gsh2 mutant, $D b \times 1$ is ectopically expressed in the LGE. C, This is also the case in the Gsh2/T/x double mutant; however, the number of cells expressing $D b \times 1$ is drastically reduced. Note that, although Dbx1 is expressed in the LGE-cortex angle in the wild type, this area lacks expression of Dbx 1 in the Gsh2 mutant and Gsh2/TIx double-mutant telencephalon. Another marker of the ventral pallium, SFRP2, is expressed in the LGE-cortex angle at E12.5 (arrow in D). At this stage, the location of the SFRP2 expression domain is shifted ventrally in Gsh2 mutants (open arrow) compared with its normal position (arrow). F, In the Gsh2/TIx double mutant, SFRP2 expression is missing. G, Also at E14.5, SFRP2 expression is detected in the LGE-cortex angle (arrow in G). $H$, After the recovery, which is occurring in Gsh2 mutants by E14.5, SFRP2 expression is undistinguishable from the wild type. I, However, in a Gsh2/TIX double mutant, SFRP2 expression is still missing. K0, Knock-out.

tity by virtue of it repressive actions on Pax6, Ngn2, and Dbx1 expression, $T l x$ is required to confine Gsh2 expression to the dorsal LGE as well as to promote the expression of $D b x 1$ and SFRP2. As we showed previously (Stenman et al., 2003b), Tlx performs these functions in concert with the pallial-enriched Pax6 gene.

\section{References}

Briscoe J, Ericson J (2001) Specification of neuronal fates in the ventral neural tube. Curr Opin Neurobiol 11:43-49.

Corbin JG, Gaiano N, Machold RP, Langston A, Fishell G (2000) The Gsh2 homeodomain gene controls multiple aspects of telencephalic development. Development 127:5007-5020.

Deacon TW, Pakzaban P, Isacson O (1994) The lateral ganglionic eminence is the origin of cells committed to striatal phenotypes: neural transplantation and developmental evidence. Brain Res 668:211-219.

Fernandez AS, Pieau C, Reperant J, Boncinelli E, Wassef M (1998) Expression of the Emx-1 and Dlx-1 homeobox genes define three molecularly distinct domains in the telencephalon of mouse, chick, turtle and frog empattern and neuronal fate in the ventral neural tube. Cell 104:861-873.

Novitch BG, Chen AI, Jessell TM (2001) Coordinate regulation of motor neuron subtype identity and pan-neuronal properties by the bHLH repressor Olig2. Neuron 31:773-789.

Olsson M, Campbell K, Wictorin K, Bjorklund A (1995) Projection neurons in fetal striatal transplants are predominantly derived from the lateral ganglionic eminence. Neuroscience 69:1169-1182.

Olsson M, Campbell K, Turnbull DH (1997) Specification of mouse telencephalic and mid-hindbrain progenitors following heterotopic ultrasound-guided embryonic transplantation. Neuron 19:761-772.

Olsson M, Bjorklund A, Campbell K (1998) Early specification of striatal projection neurons and interneuronal subtypes in the lateral and medial ganglionic eminence. Neuroscience 84:867-876.

Puelles L, Kuwana E, Puelles E, Rubenstein JL (1999) Comparison of the mammalian and avian telencephalon from the perspective of gene expression data. Eur J Morphol 37:139-150.

Puelles L, Kuwana E, Puelles E, Bulfone A, Shimamura K, Keleher J, Smiga S, Rubenstein JL (2000) Pallial and subpallial derivatives in the embryonic chick and mouse telencephalon, traced by the expression of the genes Dlx-2, Emx-1, Nkx2.1, Pax-6, and Tbr-1. J Comp Neurol 424:409-438.

Rallu M, Corbin JG, Fishell G (2002) Parsing the prosencephalon. Nat Rev Neurosci 3:943-951.

Roy K, Thiels E, Monaghan AP (2002) Loss of the tailless gene affects forebrain development and
Interestingly, in the GSh2/T/x double mutant, NKX6.2 is extopically expressed at high levels throughout the LGE VZ up to the LGE-pallium angle. J, PAX6 is expressed in the pallium at E14.5. K, In Gsh2 mutants, a recovery is occurring by E14.5, and PAX6 expression is primarily normalized. L, This recovery also occurs in Gsh2/TIx double mutants. Note that the expression domains of PAX6 and NKX6.2 nearly abut in the double mutants. (I, L). KO, Knockout; Stm, striatum. tional repression establishes progenitor cell

bryos: implications for the evolution of telence-
phalic subdivisions in amniotes. Development oster GA, Schultzberg M, Hokfelt T, Goldstein Greengard P (1987) Development of monophosphate-regulated phosphoprotein vous system, and its relationship to the arrival of presumptive dopaminergic innervation. erfen CR, Baimbridge KG, Miller JJ (1985) The tion of calcium-binding protein and parvalbu$\mathrm{min}$ in the basal ganglia of the rat and monkey. Natl Acad Sci USA 82:8780-8784. spinal cord: inductive signals and transcriptional codes. Nat Rev Genet 1:20-29. muro I, Schalling M, Jahn L, Bodmer R, Jenkins novel murine homeobox-containing gene, expressed specifically in glial cells of the brain repressor activity in vitro for a seruminducible promoter. EMBO J 12:1387-1401. In: Mouse development (Rossant J, Tam PP, onaghan AP, Grau E, Bock D, Schutz G (1995) The mouse homolog of the orphan nuclear receptor tailless is expressed in the developing Monaghan AP, Bock D, Gass P, Schwager A, tive limbic system in mice lacking the tailless gene. Nature 390:515-517.

Muhr J, Andersson E, Persson M, Jessell TM, Ericson J (2001) Groucho-mediated transcrip- 

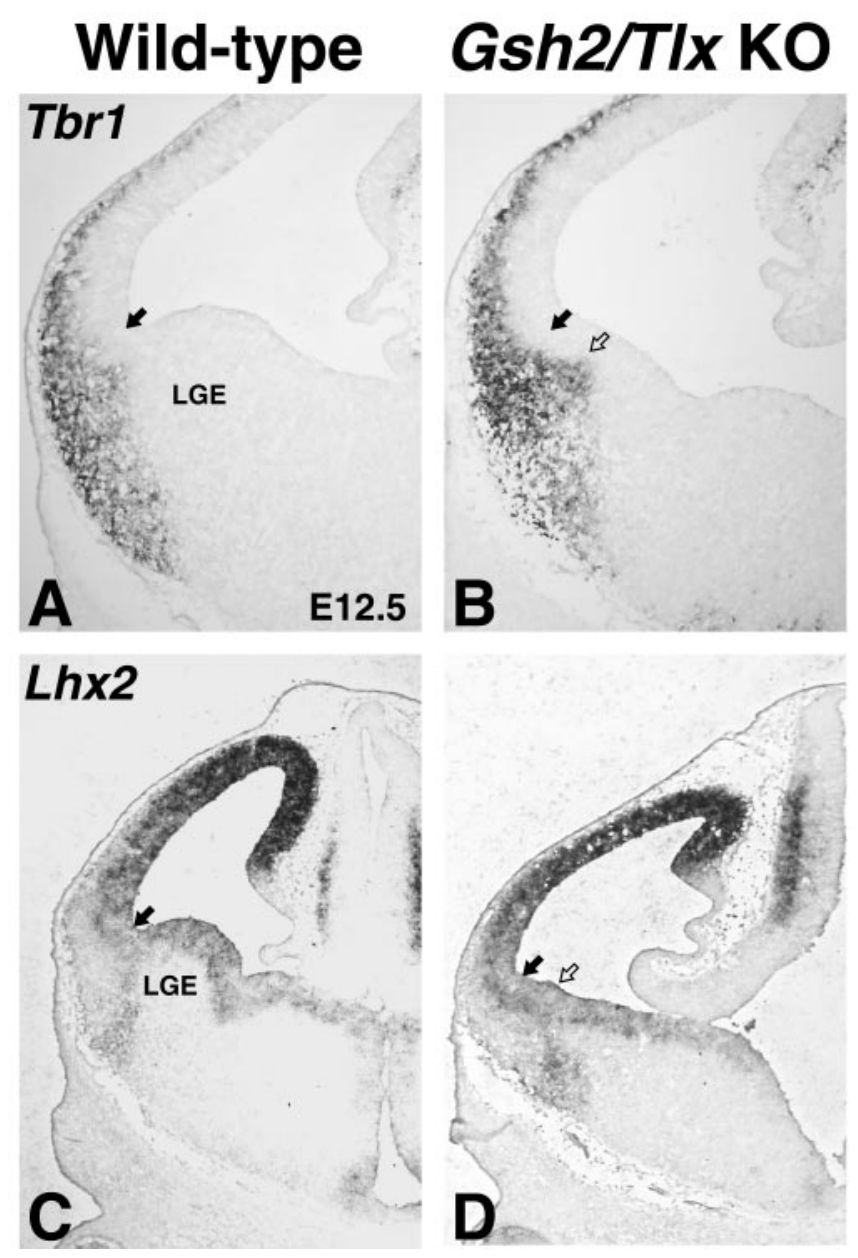

Figure 8. Some aspects of ventral pallial identity are retained even in the absence of $T / x$ and Gsh2. $A, B$, In a wild type, $\operatorname{Tbr} 1(A)$ and $L h \times 2(C)$ are expressed in the entire pallial SVZ and the ventral pallium SVZ, respectively. B, In Gsh2/T/x double mutants, SVZ expression of Tbr1 is expanded into the LGE, showing that not only the VZ adopts a pallial identity. D, In addition, Lhx2 expression in the SVZ is shifted into the LGE, showing that, despite the lack of SFRP2 and $D b \times 1$ in the VZ, aspects of ventral pallial identity are still retained in the double mutants. Filled arrows mark the pallio-subpallial boundary in wild types. Open arrows mark the ventral shift of this boundary in the double mutants. K0, Knock-out.

emotional behavior. Physiol Behav 77:595-600.

Rubenstein JL, Shimamura K, Martinez S, Puelles L (1998) Regionalization of the prosencephalic neural plate. Annu Rev Neurosci 21:445-477.
Schlüter C, Duchrow M, Wohlenberg C, Becker MH, Key G, Flad HD, Gerdes J (1993) The cell proliferation-associated antigen of antibody Ki-67: a very large, ubiquitous nuclear protein with numerous repeated elements, representing a new kind of cell cycle-maintaining proteins. J Cell Biol 123:513-522.

Schuurmans C, Guillemot F (2002) Molecular mechanisms underlying cell fate specification in the developing telencephalon. Curr Opin Neurobiol $12: 26-34$

Sommer L, Ma Q, Anderson DJ (1996) neurogenins, a novel family of atonal-related bHLH transcription factors, are putative mammalian neuronal determination genes that reveal progenitor cell heterogeneity in the developing CNS and PNS. Mol Cell Neurosci 8:221-241.

Stenman J, Toresson H, Campbell K (2003a) Identification of two distinct progenitor populations in the lateral ganglionic eminence: implications for striatal and olfactory bulb neurogenesis. J Neurosci 23:167-174.

Stenman J, Yu RT, Evans RM, Campbell K (2003b) Tlx and Pax6 co-operate genetically to establish the pallio-subpallial boundary in the embryonic mouse telencephalon. Development 130:1113-1122.

Szucsik JC, Witte DP, Li H, Pixley SK, Small KM, Potter SS (1997) Altered forebrain and hindbrain development in mice mutant for the Gsh-2 homeobox gene. Dev Biol 191:230-242.

Toresson H, Campbell K (2001) A role for Gsh1 in the developing striatum and olfactory bulb of Gsh2 mutant mice. Development 128:4769-4780.

Toresson H, Mata de Urquiza A, Fagerstrom C, Perlmann T, Campbell K (1999) Retinoids are produced by glia in the lateral ganglionic eminence and regulate striatal neuron differentiation. Development 126:1317-1326.

Toresson H, Potter SS, Campbell K (2000) Genetic control of dorsal-ventral identity in the telencephalon: opposing roles for Pax6 and Gsh2. Development 127:4361-4371.

Vallstedt A, Muhr J, Pattyn A, Pierani A, Mendelsohn M, Sander M, Jessell TM, Ericson J (2001) Different levels of repressor activity assign redundant and specific roles to Nkx6 genes in motor neuron and interneuron specification. Neuron 31:743-755.

Wang HF, Liu FC (2001) Developmental restriction of the LIM homeodomain transcription factor Islet- 1 expression to cholinergic neurons in the rat striatum. Neuroscience 103:999-1016.

Wichterle H, Garcia-Verdugo JM, Herrera DG, Alvarez-Buylla A (1999) Young neurons from medial ganglionic eminence disperse in adult and embryonic brain. Nat Neurosci 2:461-466.

Wichterle H, Turnbull DH, Nery S, Fishell G, Alvarez-Buylla A (2001) In utero fate mapping reveals distinct migratory pathways and fates of neurons born in the mammalian basal forebrain. Development 128:3759-3771.

Yu RT, Chiang MY, Tanabe T, Kobayashi M, Yasuda K, Evans RM, Umesono K (2000) The orphan nuclear receptor Tlx regulates Pax2 and is essential for vision. Proc Natl Acad Sci USA 97:2621-2625.

Yun K, Potter S, Rubenstein JL (2001) Gsh2 and Pax6 play complementary roles in dorsoventral patterning of the mammalian telencephalon. Development 128:193-205. 